\title{
MiR-433-3p Inhibits Proliferation and Invasion of Esophageal Squamous Cell Carcinoma by Targeting GRB2
}

\author{
Qizhong Shia,b Yongling Wang ${ }^{c}$ Yonghui Mu ${ }^{c}$ Xin Wang ${ }^{a} \quad$ Qingxia Fan ${ }^{a}$ \\ aDepartment of Oncology, The First Affiliated Hospital of Zhengzhou University, Zhengzhou, \\ ${ }^{b}$ Department of cardiothoracic Surgery, The Third Affiliated Hospital of Xinxiang Medical University, \\ Xinxiang, 'Department of Pathophysiology, Xinxiang Medical University, Xinxiang, China
}

\section{Key Words}

Mir-433-3p • Esophageal squamous cell carcinoma • GRB2 • Proliferation • Invasion

\begin{abstract}
Background/Aims: MicroRNAs (miRNAs) are non-coding single stranded RNAs of 17-25 nucleotides in size, and their altered expression has been observed in various cancers. Previous studies have confirmed that miR-433-3p has effects on cancer cell proliferation, invasion, and migration, and its expression also correlates with sensitivity to chemotherapy. However, to date, there have been no studies on the biological functions of miR-433-3p in esophageal squamous cell carcinoma (ESCC). Methods: The Cell Counting Kit-8, transwell, and matrigel assays were used to test the effects of miR-433-3p and its predicted target, growth factor receptor-bound protein 2 (GRB2), on the proliferation, migration, and invasion of Eca109 and KYSE30 cells, two types of esophageal cancer cell lines. The miR-433-3p binding site in the $3^{\prime}$ untranslated region (UTR) region of GRB2 was predicted and verified using miRNA target site prediction software and structuring correct mutant examination. Western blotting and fluorescent quantitative PCR (FQ-PCR) techniques were employed to evaluate GRB2 expression. The inhibitory effects of miR-433-3p on tumor growth were investigated using a tumor xenograft model. Results: The binding site of miR-433-3p was identified in the 3'UTR region of GRB2. Western blotting and FQ-PCR showed that miR-433-3p inhibited the mRNA and protein expression of GRB2. Overexpression of GRB2 inhibited tumorigenesis in nude mice. MiR-433-3p overexpression inhibited the proliferation, migration, and invasion of ESCC cells by suppressing GRB2 gene expression. Conclusions: Our findings suggest that targeting miR-433-3p may have therapeutic benefits in ESCC.

(C) 2018 The Author(s) Published by S. Karger AG, Basel
\end{abstract}

\section{Introduction}

Esophageal cancer is a malignant tumor of the esophagus with high morbidity and mortality. It is mainly divided into esophageal squamous cell carcinoma (ESCC), the most 
Shi et al.: MiR-433-3p Inhibits Proliferation and Invasion of Esophageal Squamous Cell Carcinoma by Targeting GRB2

common esophageal cancer worldwide, and adenocarcinoma [1, 2]. ESCC predominantly occurs in East Asia, where it accounts for more than $90 \%$ of esophageal cancer cases $[3,4]$. Despite great efforts made in the past decade, the mechanisms underlying the metastasis of cancer cells are not fully understood.

MicroRNAs (miRNAs) are non-coding single stranded RNAs of 17-25 nucleotides (nt) in size, and their altered expression has been found in various cancers. Recent studies have shown that miR-433-3p, a tumor suppressor located on chromosome 12, inhibited the proliferation, invasion, and drug resistance of various tumor cells, and repressed the growth of hepatocellular carcinoma cells [5, 6]. Yang et al. that miR-433-3p blocked the invasion of liver cancer cells [7]. In a study of gallbladder cancer, miR-433-3p also blocked the invasion of gallbladder cancer cells by repressing the epithelial-mesenchymal transition [8]. In cervical cancer, miR-433-3p prevented fluorouracil drug resistance in cervical cancer cells [9]. However, there have been no studies on the biological functions of miR-433-3p in ESCC. Our previous study showed that miR-433-3p is negatively correlated with lymphatic metastasis and tumor growth of ESCC and is positively correlated with lymphocyte differentiation.

This study investigated the effects of miR-433-3p and its predicted target, growth factor receptor-bound protein 2 (GRB2), on the proliferation and invasion of ESCC cell lines, Eca109 and KYSE30, transfected with miR-433-3p mimics. In addition, the binding site of miR-43330 on GRB2 was identified.

\section{Materials and Methods}

\section{Materials}

Eca109 and KYSE30 ESCC cells were purchased from American Type Culture Collection (Manassas, VA, USA). Female nude mice (3-4 weeks of age, 16-18 g) were purchased from the Experimental Animal Institute of the Chinese Academy of Medical Sciences (Animal Production License: Jing No. SCXK 2009-0008; Beijing, China). MiR-433-3p-mimics were from Biao Maike Biotechnology Co., Ltd. (City, China); Lipofectamine 3000 and RPMI-1640 medium were from Thermo Fisher Scientific (Waltham, MA, USA); the CCK-8 Kit was purchased from YiSheng Biotechnology Co., Ltd. (Shanghai, China); Matrigel was from SaiTai Biotechnology Co., Ltd. (City, China); fetal bovine serum (FBS) was obtained from Sijiqing Bioengineering Material Co., Ltd. (Hangzhou, China); dimethyl sulfoxide was from Sigma (St. Louis, MO, USA); green streptomycin was from Southern Pharmaceutical Co., Ltd. (City, China); trypsin was from Ruiqi Biology Co., Ltd. (Shanghai, China); 24-well Transwell chambers were purchased from Corning Corporation (Corning, NY, USA); Sal I, Xho I, and T4 DNA ligase was from New England Biolabs (Ipswich, MA, USA); GoTaq® Green Master Mix, pGEM-T Easy Vector Systems, agarose, dNTPs (100 mM), and Dual-Luciferase® Reporter Assay System were purchased from Promega (Fitchburg, WI, USA); Plasmid Mini Kit, Gel DNA Recovery Kit, and 30\% acrylamide were from Kangwei Century Biotechnology Co. (Beijing, China); the HiSpeed Plasmid Maxi Kit was from QIAGEN (Gaithersburg, MA, USA); Trizol Reagent was from Invitrogen (Carlsbad, CA, USA); LHC9 Medium was from GIBCO (Gaithersburg, MA, USA); Easy Mutagenesis System was obtained from Golden Biotechnology Co., Ltd. (Beijing, China); Reverse Transcription Kit, 10× Loading Buffer, and the SYBR ${ }$ PrimeScript $®$ RT-PCR Kit were from TaKaRa Biotechnology (Dalian, China); DNA Marker I/II/III/IV was from TIANGEN Biotech Co. (Beijing, China); TaqMan Small RNA Assay was from ABI (Sacramento, CA, USA); tryptone and yeast extract were purchased from OXOID (City, UK); Tris base was from Amersco (Framingham, MA, USA); Triton X-100 was from Beijing Chemical Company (Beijing, China); and IPTG and X-gal were purchased from Amersco (Framingham, MA, USA).

\section{Cell culture and transfection}

HEEC, Eca109, and KYSE30 cells were cultured in RPMI-1640 medium containing 10\% FBS and placed in the incubator at $37^{\circ} \mathrm{C}$ and $5 \% \mathrm{CO}_{2}$. At $24 \mathrm{~h}$ before transfection, they were inoculated into 6-well plates at a density of $4 \times 10^{5}$ cells/well. At about $80 \%$ confluence, the cells were transfected with miR-433-3p-mimics, scrambled miRNA, pcDNA3-GRB2, miR-433-3p-mimics + pcDNA3-GRB2, pmirGLO-GRB2 3'UTR + miR-4333p-mimics, pmirGLO-GRB2 3'UTR mutant + miR-433-3p-mimics, or the negative control. The cells were further cultured in the incubator $\left(37^{\circ} \mathrm{C}\right.$ and $\left.5 \% \mathrm{CO}_{2}\right)$ to observe changes in proliferation and invasion, and also for the luciferase assay. 


\section{Cellular Physiology Cell Physiol Biochem 2018;46:2187-2196 \\ \begin{tabular}{l|l} 
and Biochemistry Published online: May 04, 2018 & $\begin{array}{l}\text { () 2018 The Author(s). Published by S. Karger AG, Basel } \\
\text { www.karger.com/cpb }\end{array}$
\end{tabular}}

Shi et al.: MiR-433-3p Inhibits Proliferation and Invasion of Esophageal Squamous Cell

Carcinoma by Targeting GRB2

\section{CCK-8 assay}

After transfection with the constructs noted above, the cells were inoculated into 96-well plates (1500 cells per well); experiments were performed in triplicate. A total of $10 \mu \mathrm{L}$ CCK-8 solution was added to each well. The optical density (OD) value was measured by a microplate reader at $450 \mathrm{~nm}$ (three times total in 6 days). Cell viability probability (\%) = (assay well OD - empty group well OD)/(control well OD - empty group well OD) $\times 100 \%$. According to the cell viability probability, the inhibition curve was drawn and the $\mathrm{IC}_{50}$ value was calculated.

\section{Transwell and matrigel invasion assays}

When the cells reached a density of $5 \times 10^{4}$ per well, they were transfected with the constructs described above. The cells in each well were added to the top chamber of the Transwell, and $600 \mu \mathrm{L}$ culture medium containing 10\% FBS was added to the bottom chamber. Then the Transwells were placed in the incubator $\left(37^{\circ} \mathrm{C}, 5 \% \mathrm{CO}_{2}\right)$. After $24 \mathrm{~h}$, a cotton swab was used to gently wipe the non-invasive cells in the top chamber. The cells that migrated to the bottom chamber were fixed in $4 \%$ paraformaldehyde and dyed with $0.25 \%$ crystal violet. The average number of cells from five randomly selected five fields were counted under a microscope at $200 \times$. Subsequent invasion experiments were performed similarly as the Transwell assay with the exception that Matrigel was added to the chambers.

\section{Nude mice assay}

The nude mice were fed in the ultra-clean biological laminar flow rack. The feed, mat, and drinking water were sterilized by high pressure and replaced every 3 days. Twelve nude mice were randomly divided into two groups: negative control group (injected with tumor cells transfected with miR-433-3p-mimics) and mimic group (injected with tumor cells transfected with miR-433-3p-mimics). Six mice in each group were fed in different cages. Cells were collected and made into cell suspensions in the logarithmic growth phase. The subcutis in the right lateral upper arm of each nude mice was injected with $2 \times 10^{6}$ Eca109 cells (volume, $0.1 \mathrm{~mL}$ ). On day 6 post-injection, new organisms appear at the inoculation site. The tumor volume was measured, including the Long diameter (L) and short diameter (S), with a caliper every 5 days using the formula: volume $=$ length $\times$ width ${ }^{2} / 2$ at the end of a 36-day observation period.

\section{Western blot}

Western blotting was performed according to the procedure outlined in [8]. Briefly, the protein was extracted after $48 \mathrm{~h}$ and separated by 10\% SDS-PAGE. Then the proteins were electrotransferred to the membrane and blocked in blocking buffer. The membranes were incubated with anti-GRB2 $(1: 1,000)$ and anti-II $(1: 1,000)$ primary antibodies. Proteins were visualized by ECL luminescence detection; $\beta$-actin was used as the loading control. Densitometry was performed using Quantity One software.

\section{FQ-PCR}

Total RNA was extracted using the Trizol reagent. Using the SYBR® PrimeScript $₫$ RT-PCR Kit, singlestranded cDNA was obtained by reverse transcription. The FQ-PCR instrument (50-811 Tgradient96) was used to quantitatively test by the two-step method. Using the $\Delta \Delta \mathrm{CT}$ method, CT values were recorded and analyzed.

\section{Cloning and vector construction}

Total RNA was extracted from cells in the logarithmic growth phase with Trizol reagent. The first cDNA-strand was synthesized by reverse transcription and PCR was used to amplify the cDNA of GRB2. The following GRB2 primers were used: 5' CACAGACCGGTATGGAAGCCATCGCCAA3' (upstream), 5' CACAGGAATTCTTAGACGTTCCGGTTCACTG3' (downstream). The PCR product was cloned into the pGEM®-T easy vector. The reaction was conducted at $12^{\circ} \mathrm{C}$ for $12 \mathrm{~h}$ and then at $65^{\circ} \mathrm{C}$ for $10 \mathrm{~min}$. The product was transformed into DH5 $\alpha$ competent cells, which were screened on agar plates containing ampicillin. The pGEMT-GRB2 vector was identified by enzyme digestion and sequencing. Sangon Biotech Co. (Shanghai, China) synthesized the sequence from $2117 \mathrm{nt}$ to $2164 \mathrm{nt}$ downstream of the GRB2 stop codon, which contained the predicted binding site of miR-433-3p. The XhoI enzyme restriction site was designed at the $5^{\prime}$ end and the Sall enzyme restriction site was designed at the $3^{\prime}$ end. The Xhol and Sall enzymes were used to digest the pmirGLO Dual-Luciferase miRNA Target Expression Vector and GRB2 3'UTR was ligated into 
Shi et al.: MiR-433-3p Inhibits Proliferation and Invasion of Esophageal Squamous Cell Carcinoma by Targeting GRB2

the vector, which was used to construct the pmirGLO-GRB 2 3'UTR expression vector. The pmirGLO-GRB 2 3'UTR expression vector was transformed into DH5 $\alpha$ competent cells. The pmirGLO-GRB2 3'UTR was identified by restriction digest and sequencing. The pcDNA3-GRB2 expression vector was constructed by the same process and verification steps. Eca109 and KYSE30 cells were co-transfected with miR-433-3pmimics and pcDNA3-GRB2 expression vector. Cell proliferation and invasion were measured on days 1-6 post-transfection.

Construction of the PmirGLO-GRB2-3'UTR mutant followed by the luciferase reporter assay

Construction of the PmirGLO-GRB2-3'UTR mutant was performed by the BoShang Sequencing Company (Shanghai, China). Vector NTI 11.0 software was used to make comparisons between the mutant and wild-type. The primers used were PmirGLO-GRB2-3'UTRWT: $\quad 5^{\prime} \quad$ GAGCTCTTATTTTGGAATTGGTGTATATCATGAAGCCTTGCTGAACTAAGTTTTCTCGAG $3^{\prime}$. PmirGLO-GRB2-3'UTR-Mutant (The mutation site is underlined): $5^{\prime}$ GAGCTCTTATTTTGGAATTGGTGTATTAGTACTTGCCTTGCTGAACTAAGTTTTCTCGAG 3'. The PmirGLO-GRB2 3'UTR and pmirGLO-GRB2 3'UTR mutant were verified by sequencing. KYSE30 cells were co-transfected with miR-433-3p and pmirGLO-GRB2 3'UTR or with miR-433-3p and pmirGLO-GRB2 3'UTR mutant. After $48 \mathrm{~h}$, the cells were tested using the Dual-Luciferase Reporter Assay System Kit. Twenty microliters lysate solution was added to each well of the 96-well white plate. Then, $5 \mu \mathrm{L}$ LAR II was added to detect luciferase activity. Finally, $50 \mu \mathrm{L}$ Stop \& Glo was added to terminate the luciferase reaction and activate the Renilla luciferase reaction. The amount of Renilla luciferase activity was measured.

\section{Statistical analysis}

The data from each group are presented as the mean \pm standard deviation and were analyzed using SPSS 11.0 statistical software SPSS (Chicago, IL, USA). The homogeneity of variance analysis and normal distribution test were performed before statistical analysis. Statistical analysis was performed using the paired $t$-test or single-factor variance analysis. When there was non-homogeneity variance or non-normal distribution, the variable was changed and the homogeneity of variance analysis and normal distribution test were performed. If there was still non-homogeneity variance or non-normal distribution, the nonparametric test was conducted. P values less than 0.05 were considered statistically significant.

\section{Results}

\section{MiR-433-3p inhibited the proliferation of Eca109 and KYSE30 cells}

We analyzed the effects of miR-433-3p on cell proliferation by transfecting cells with miR-433-3p-mimics and performing Cell Counting Kit-8 (CCK-8) cell viability assays. Compared with transfections with a negative control and scrambled vector, transfection with miR-433-3p-mimics did not significantly change the rate of cell proliferation on days 1 and 2 post-transfection ( $p>0.05$, Fig. 1A). However, on days 3-6 post-transfection, the proliferation of miR-433-3p mimic-transfected cells was markedly lower than that in cells transfected with the negative control $(p<0.05)$; there was no significant difference in proliferation between the scrambled group and negative control group ( $p>0.05$, Fig. $1 \mathrm{~A}, \mathrm{~B})$. These results indicated that miR-433-3p suppressed the proliferation of Eca109 and KYSE30 cells. In nude mice assays, the volume of metastatic tumors in miR-433-3p-mimics group was significantly smaller than that in the negative control group $(p<0.05)$, which confirmed the abovementioned observations (Fig. 1C, D).

\section{MiR-433-3p suppressed the invasion and migration of Eca109 and KYSE30 cells}

Transwell and matrigel assays were used to measure the effects of miR-433-3p on the invasion and migration of Eca109 and KYSE30 cells. Cells transfected with miR-433-3p mimics had significantly less invasion and migration capabilities than those transfected with the negative control or scrambled miRNA ( $p<0.05$, Figs. $2 \mathrm{~A}, 2 \mathrm{~B}, 3 \mathrm{~A}, 3 \mathrm{~B})$; there were no significant differences between the negative control and scrambled groups $(p>0.05)$. These results indicated that miR-433-3p suppressed the invasion and migration of ESCC cells. 
Fig. 1. MiR-433-3p inhibited the proliferation of Eca109 and KYSE30 cells. The optical density value of Eca109 (A) and KYSE30 (B) cells transfected with miR-433$3 \mathrm{p}$ mimics, the negative control, and scrambled miRNAs was used to evaluate cell proliferation. (C) Xenograft experiments were performed to confirm the effects of miR-433-3p on tumor growth in vivo. Tumor volume was measured with a caliper every 6 days using the formula: volume = length $\times$ width $^{2} / 2$. (D) Photographs of xenograft tumors after the 36-day observation period. All experiments were repeated three times. All data are shown as the mean \pm standard deviation (SD). ${ }^{*} \mathrm{p}<0.05, \quad{ }^{*} \mathrm{p}<0.01$ versus the negative control; ${ }^{\#} \mathrm{p}<0.05$, ${ }^{\# \#} \mathrm{P}<0.01$ versus scrambled miRNA.

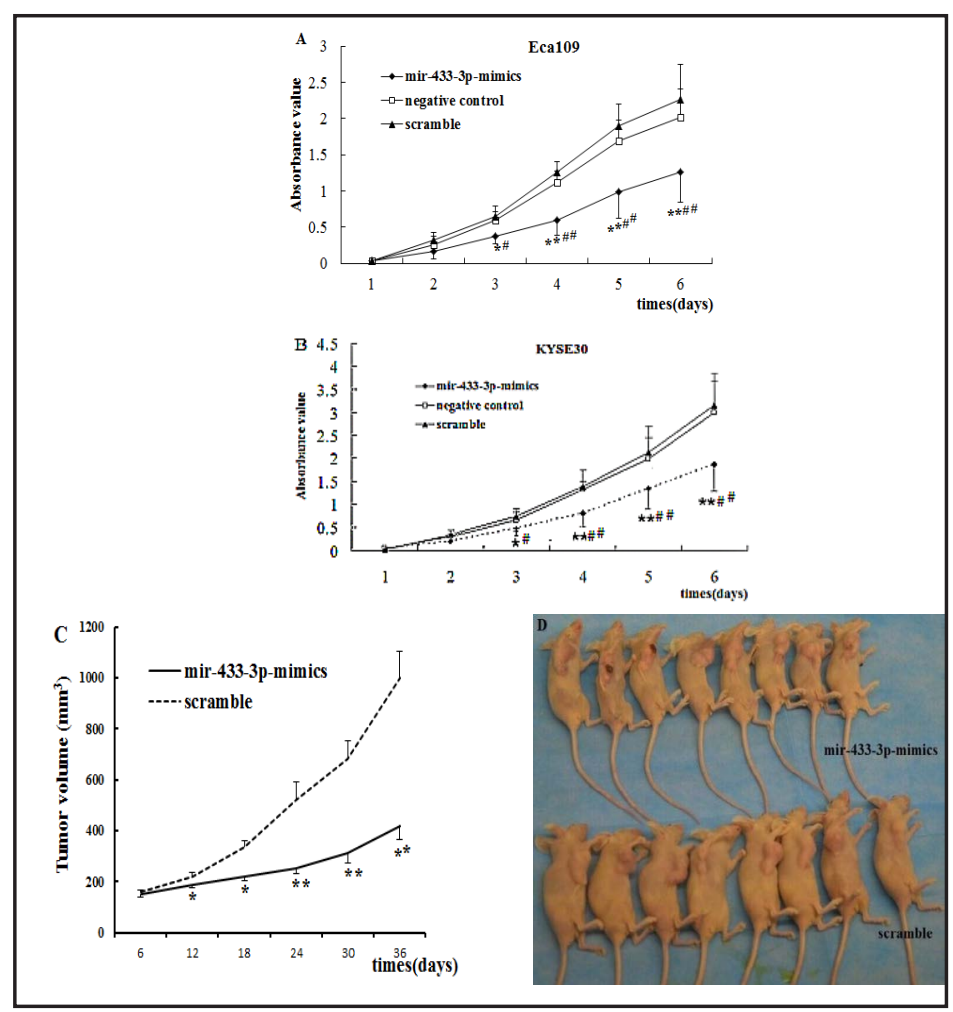

Fig. 2. MiR-433-3p suppressed the migration of Eca109 (A) and KYSE30 cells (B). Mean cell number in five randomly selected visual fields in the migration and transwell assays. All experiments were repeated three times. All data are shown as the mean \pm SD. ${ }^{*} \mathrm{p}<0.05$ versus the negative control; ${ }^{\mathrm{p}}<0.05$ versus scrambled miRNA.

The binding site of miR-433-3p was identified in the $3^{\prime}$ untranslated region of GRB2

We used miRNA target prediction software programs, namely, miRnada, Pictar2, RNA22, RNAhybrid, and Targetscan, to predict the binding site of miR-433-3p on GRB2. All programs predicted that the miR-433-3p binding site was in the $3^{\prime}$ untranslated region (UTR) of GRB2, downstream of the stop codon at 2137 nt. The programs also predicted an 8-mer site (Fig. 4).

\section{Luciferase reporter assay verified the miR-433-3p binding site}

We constructed a GRB2 3'UTR mutant, encompassing region 2130-2150 nt, to verify the predicted miR-433-3p binding site. T3'he mutant was inserted into the pmirGLO DualLuciferase miRNA Target Expression Vector. After the sequences are confirmed, KYSE30 cells were co-transfected with pmirGLO-GRB2 $3^{\prime}$ UTR-Wt and miR-433-3p-mimics or pmirGLOGRB2 3'UTR-mut and miR-433-3p mimics. The results of the luciferase assay showed that miR-433-3p markedly inhibited the luciferase activity of the pmirGLO-GRB2 3'UTR-Wt group compared with the pmirGLO-GRB2 $3^{\prime} \mathrm{UTR}$-mut and negative control groups $(p<0.05$; Fig. 5), but no significant difference in luciferase activity was found between the pmirGLOGRB2 3'UTR-mut and negative control groups ( $p<0.05$; Fig. 5). 


\section{MiR-433-3p inhibited the mRNA and protein expression of GRB2}

The results of the fluorescence quantitative PCR (FQ-PCR) assay showed that the expression of miR-433-3p mRNA significantly decreased and the expression of GRB2 mRNA significantly increased in Eca109 and KYSE30 cells compared with HECC $(p<0.05)$. Western blot analysis showed that the levels of GRB2 protein in Eca109 and KYSE30 cells were significantly higher than those in $\operatorname{HEEC}(p<0.05$; Fig. $6 \mathrm{~A})$. The mRNA and protein expression of GRB2 in Eca109 and KYSE30 cells transfected with miR-433-3p mimics was significantly lower than that in the negative control group $(p<0.05)$; there was no significant difference between the negative control and scrambled miRNA groups (Fig. 6B). These results suggested that miR-433-3p inhibited the mRNA and protein expression of GRB2.

\section{GRB2 increased the proliferation, migration, and invasion of ESCC cells}

Eca109 and KYSE30 cells were transfected with pcDNA3-GRB2 vector to further demonstrate that GRB2 can increase the proliferation of ESCC cells. The results showed that the proliferation of pcDNA3-GRB2 cells significantly increased compared to cells transfected with pcDNA3 (empty vector) on days 5 and 6 post-transfection $(p<0.05)$. In addition more cells in the pcDNA3-GRB2 group had migratory and invasive abilities than those in the pcDNA3 group ( $p<0.05$; Fig. 7A-F).

\section{MiR-433-3p inhibited the proliferation, migration, and invasion of ESCC cells by targeting} GRB2

In Eca109 and KYSE30 cells, western blot analysis showed that the protein expression of GRB2 in cells transfected with miR-433-3p-mimics + pcDNA3 was significantly lower than that in cells transfected with miR-433-3p-mimics + pcDNA3-GRB2 $(p<0.05)$. The cell proliferation assay showed that the proliferation of cells transfected with miR-433-3p-

Fig. 3. MiR-433-3p suppressed the invasion of Eca109 (A) and KYSE30 cells (B). Mean cell number of five randomly selected visual fields in the invasion assay. $* \mathrm{p}<0.05$ versus the negative control, ${ }^{*} \mathrm{p}<0.05$ versus scrambled miRNA. All experiments were repeated three times. All data are shown as the mean \pm SD.

Fig. 4. miR-433-3p binding site is in the $3^{\prime}$ UTR of GRB2. The binding site of miR-433-3p was found to downstream of the stop codon in the GRB2 3'UTR at $2137 \mathrm{nt}$.

Fig. 5. Luciferase reporter assay verified the prediction site. MiR433-3p significantly inhibited the luciferase activity of pmirGLOGRB2 3'UTR. All experiments were repeated three times. All data are shown as the mean \pm SD. ${ }^{*} \mathrm{p}<0.05$ versus the negative control, $\# \mathrm{p}<0.05$ versus the empty vector.

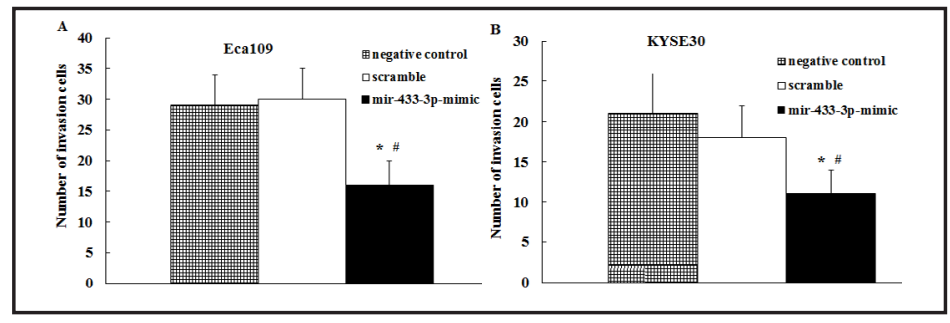

3' uguggcuCCUCGGGUAGUACUa 5' hsa-miR-433

| | || ||

5' uggaauugGUGUAUAUCAUGAa 3' GRB2

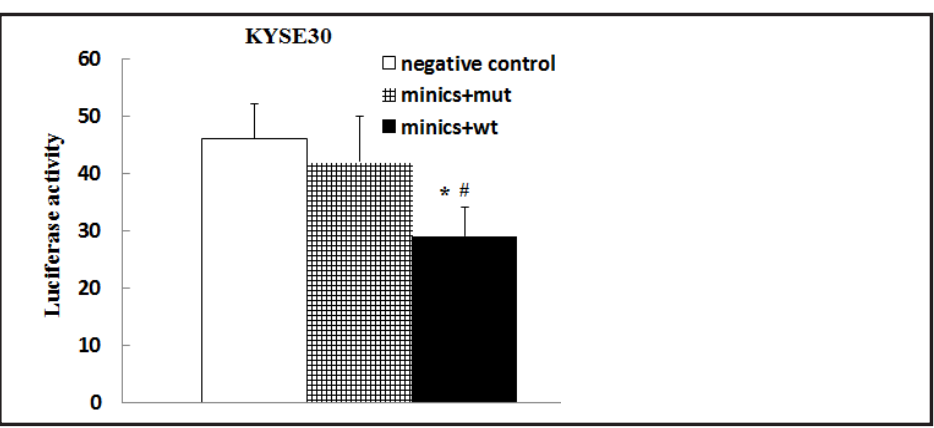


Fig. 6. MiR-433-3p inhibited the protein expression of GRB2. (A) Quantitative real-time PCR (qRTPCR) was performed in HECC, Eca109, and KYSE30 cells; (B) Western blotting was used to detect GRB2 protein levels in Eca109 and KYSE30 cells overexpressing miR433-3p ( ${ }^{*} \mathrm{p}<0.05$ versus HECC). (C) qRT-PCR was performed in Eca109 and KYSE30 cells transfected with miR-433-3p mimics or empty vector. (D) Western blotting was used to evaluate GRB2 protein expression in Eca109 and KYSE30 cells overexpressing miR-433-3p. All experiments were repeated

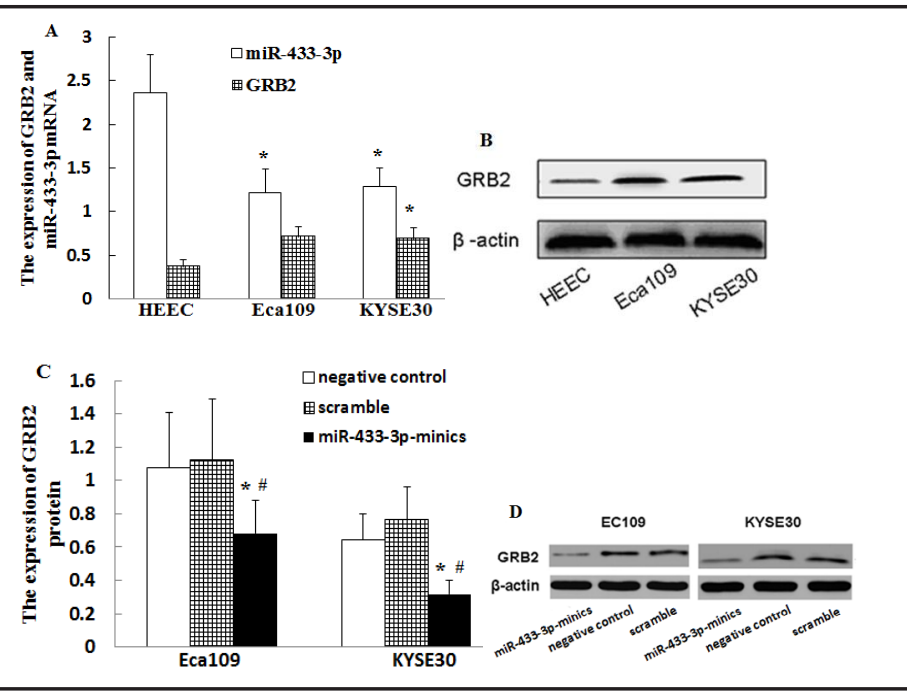
three times. All data are shown as the mean \pm SD. ${ }^{*} p<0.05$ versus negative control, ${ }^{\#} p<0.05$ versus scrambled miRNA.

Fig. 7. GRB2 increased the proliferation and invasion of ESCC cells. After transfection with pcDNA3-GRB2 expression vector, the proliferation of (A) Eca109 and (B) KYSE30 cells significantly increased compared with the pcDNA3 group. The migration of (C) Eca109 and (D) KYSE30 cells significantly increased after transfection with pcDNA3-GRB2 expression vector. The numbers of (E) Eca109 and (F) KYSE30 cells significantly increased after transfection with pcDNA3GRB2 expression vector $(* \mathrm{p}<0.05$ compared with the pcDNA3 group). All experiments were repeated three times. All data are shown as the mean \pm SD.

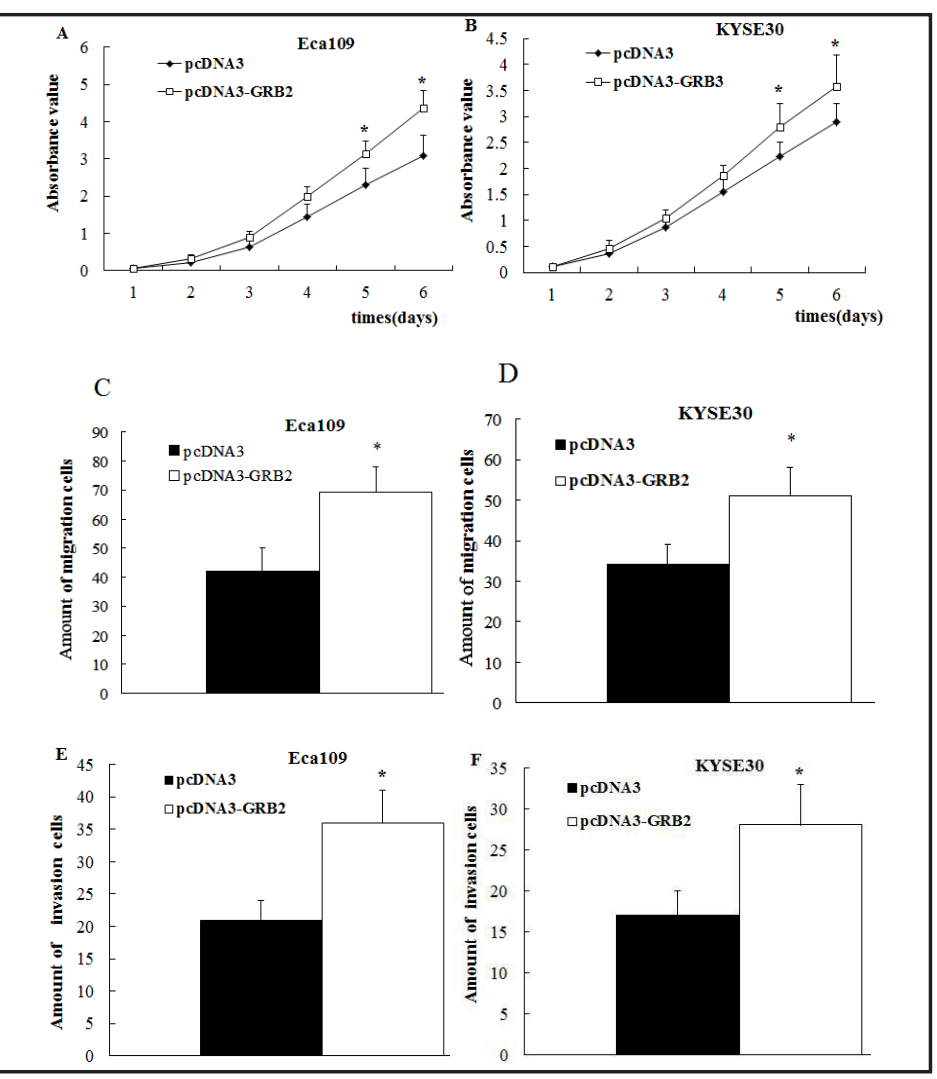

mimics + pcDNA3 was significantly lower than that of cells transfected with miR-433-3pmimics + pcDNA3-GRB2 $(p<0.05)$. The cell migration and invasion assay suggested that a higher number of cells transfected with miR-433-3p- mimics+pcDNA3 had migratory and invasive abilities compared with cells transfected with miR-433-3p- mimics + pcDNA3-GRB2 $(p<0.05$; Fig. 8A-G). These results demonstrated that miR-433-3p inhibited the proliferation, migration, and invasion of ESCC cells by suppressing GRB2 activity. 
Fig. 8. MiR-433-3p inhibited the proliferation and invasion of ESCC cells by suppressing GRB2 activity. (A) In Eca109 and KYSE30 cells, the protein expression of GRB2 in cells transfected with miR433-3p-mimics+pcDNA3 was significantly lower than that in cells transfected with miR-4333p-mimics+pcDNA3-GRB2. The proliferation ability of (B) Eca109 and $(\mathrm{C})$ KYSE30 cells in the miR433-3p-mimics + pcDNA3 group was significantly lower than that of cells in the miR-433-3p-mimics + pcDNA3-GRB2 group. The migration ability of (D) Eca109 and (E) KYSE30 cells in the miR433-3p-mimics+pcDNA3 group was significantly lower than that of cells in the miR-433-3p-mimics + pcDNA3-GRB2 group. The invasion ability of (F) Eca109 and (G) KYSE30 of cells in the miR433-3p-mimics + pcDNA3 group was significantly lower than that of cells in the miR-433-3p-mimics + pcDNA3-GRB2 group $\left({ }^{*} \mathrm{p}<0.05\right.$, $* * \mathrm{p}<0.01$ compared with the mimics + pcDNA3-GRB2 group). All experiments were repeated three times. All data are shown as the mean \pm SD.

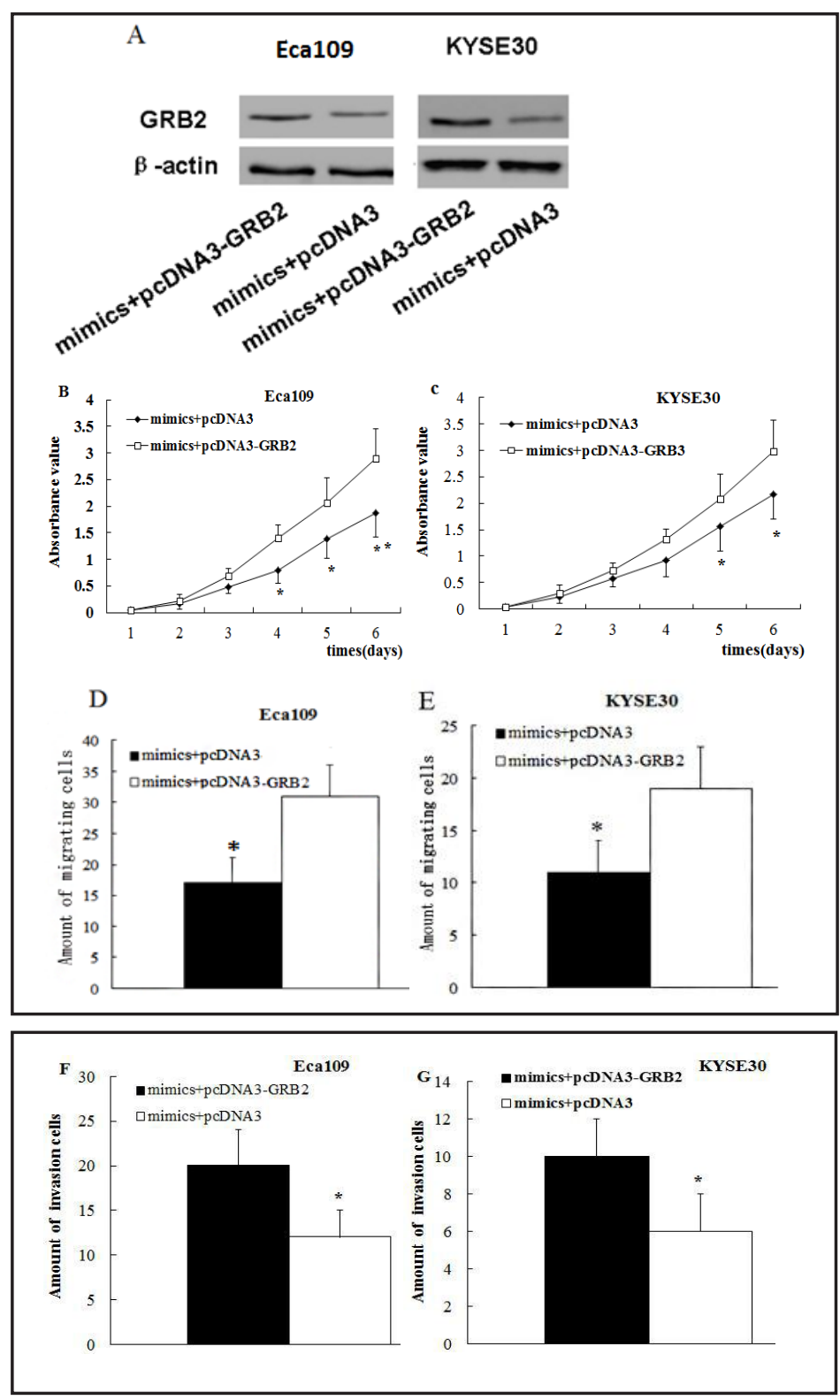

\section{Discussion}

Esophageal cancer is a malignant tumor of the esophagus with high morbidity and mortality due to recurrence, metastasis, and drug resistance. Because of its poor prognosis, the development of novel targets and more effective strategies for the treatment of this disease are urgently needed.

MiR-433-3p affects the proliferation, invasion, and migration of many types of cancer cells [10-14]; however, to date, there have been no studies on its biological functions in ESCC. Therefore, in this study, we transfected the ESCC cell lines, Eca109 and KYSE30, with miR-433-3p-mimics and found that their proliferation, invasion, and migration capabilities significantly decreased compared with the control group. These data were confirmed by nude mice tumorigenesis experiments. Furthermore, the potential binding site of miR-433-3p on GRB2, was predicted using miRNA target prediction software and verified with luciferase assays using pmirGLO-GRB2 3'UTR and pmirGLO-GRB2 3'UTR constructs. The experiments identified the binding site of miR-433-3p as being in the $3^{\prime}$ UTR of GRB2 downstream of 
the transcription stop codon at $2137 \mathrm{nt}$ in an 8-mer combination. miR-433-3p significantly inhibited the luciferase activity of pmirGLO-GRB2 $3^{\prime}$ UTR and did not significantly inhibit the luciferase activity of the pmirGLO-GRB2 3'UTR mutant. The expression of miR-433-3p mRNA significantly decreased and that of GRB2 mRNA and protein significantly increased in Eca109 and KYSE30 cells compared with HEEC cells. In addition, GRB2 mRNA and protein expression in Eca109 and KYSE30 cells significantly decreased after transfection with miR433-3p-mimics compared to cells transfected with the negative control or empty vector. The data demonstrated that miR-433-3p inhibited the expression of GRB2. We compared the cell numbers between the pcDAN3-GRB2 and pcDNA3 groups to further verify the effects of GRB2 in ESCC. To this end, we compared cells transfected with miR-433-3p-mimics + pcDNA3 with cells transfected with miR-433-3p-mimics + pcDNA3-GRB2. These results indicated that miR-433-3p inhibited the proliferation and invasion of ESCC cells by repressing GRB2 activity.

Song et al. predicted that the length of pri-mir433-3p is $1809 \mathrm{nt}$ and that of pre-mir433$3 p$ is $124 \mathrm{bp}$ [15]. Studies on gastric cancer have shown that the expression of miR-433-3p in cancer tissues is significantly lower than that in para-tumor tissue. The overexpression of miR-433-3p was shown to significantly extend the cell cycle and suppress the proliferation and migration of gastric cancer cells [16-18]. In a study on oral squamous cell carcinoma, it was found that the overexpression of miR-433-3p significantly inhibited the proliferation and invasion of oral squamous cell carcinoma cells. In addition, in a liver cancer study, miR433-3p was found to inhibit the migration of liver cancer cells. Our results are in accordance with these studies.

Bioinformatics analysis has predicted that miRNAs are involved in regulating more than $50 \%$ of protein-coding genes, and that the human nervous system can express about $70 \%$ of miRNAs. Cyclic miRNAs are derived from the active secretion of cells, the release of apoptotic or necrotic cells, and the cleavage of circulating cells. A recent study confirmed that miR433-3p can suppress the malignant behavior of glioma cells. The differential expression of miRNAs was detected in the cerebrospinal fluid and blood of patients with SZ, which suggests that miRNA may be a very promising biological marker for the treatment of mental disorders.

In conclusion, the results of our study provide a novel target for the diagnosis and treatment of ESCC. Future studies are needed to obtain more comprehensive information on the downstream signaling pathway of miR-433-3p-GRB2.

\section{Disclosure Statement}

All authors declare to have no conflict of interests.

\section{References}

$>1$ Hu HB, Jie HY, Zheng XX: Three Circulating LncRNA Predict Early Progress of EsophagealSquamous Cell Carcinoma. Cell Physiol Biochem 2016;40:117-125.

-2 Liu B, Jia Y, Cao Y, Wu S, Jiang H, Sun X, Ma J, Yin X, Mao A, ShaM: Overexpression of Phosphoserine Aminotransferase 1 (PSAT1) Predicts Poor Prognosis and Associates with Tumor Progression in Human Esophageal Squamous Cell Carcinoma.Cell Physiol Biochem 2016;39:395-406.

- 3 Pengxiang Chen, Qingxu Song, Jie Han, Huapu Xu, Tong Chen, Jiaqi Xu, Yufeng Cheng: Sitting time and occupational and recreational physical activity in relation to the risk of esophageal squamous cell carcinoma.Onco Targets Ther 2017;10:4787-4794.

4 Abnet CC, Arnold M, Wei WQ: Epidemiology of Esophageal Squamous Cell Carcinoma. Gas-troenterology 2017;S0016-5085:36039.

5 Xue J, Chen LZ, Li ZZ,Hu YY,Yan SP,Liu ly: MicroRNA-433 inhibits cell proliferation in hepatocellular carcinoma by targeting p21 activated kinase (PAK4).Mol Cell Biochem 2015;399:77-86. 
Shi et al.: MiR-433-3p Inhibits Proliferation and Invasion of Esophageal Squamous Cell Carcinoma by Targeting GRB2

6 Hu HB, Jie HY, Zheng XX: Three Circulating LncRNA Predict Early Progress of EsophagealSquamous Cell Carcinoma. Cell Physiol Biochem 2016;40:117-125.

7 Yang Z,Tsuchiya H,Zhang Y, Hartnett ME, Wang L: MicroRNA-433 inhibits liver cancer cell migration by repressing the protein expression and function of cAMP response element-binding protein.J Biol Chem 2013;288:28893-28899.

-8 Xu X,Zhu Y,Liang Z, Li S, Xu X, Wang X, Wu J, Hu Z, Meng S, Liu B, Qin J, Xie L, Zheng X : c-Met and CREB1 are involved in miR-433-mediated inhibition of the epithelial-mesenchymal transition in bladder cancer by regulating Akt/GSK-3 $\beta$ /Snail signaling.Cell Death Dis 2016;7:e2088.

-9eisuke Gotanda, Takeshi Hirota, Nozomi Matsumoto, Ichiro Leiri : MicroRNA-433 negatively regulates the expression of thymidylate synthase (TYMS) responsible for 5-fluorouracil se- nsitivity in HeLa cells.BMC Cancer 2013;13:369.

10 Sun S, Wang X, Xu X, Di H, Du J, Xu B, Wang Q Wang J: MiR-433-3p suppresses cell growth and enhances chemosensitivity by targeting CREB in human glioma.Oncotarget 2017;8:5057-5068.

11 Tang X, Lin J, Wang G, Lu J: MicroRNA-433-3p promotes osteoblast differen- tiation through targeting DKK1 expression.PLos One 2017;12:e0179860.

12 Liang C, Ding J, Yang Y, Deng L, Li X: MicroRNA-433 inhibits cervical cancer progression by directly targeting metadherin to regulate the AKT and $\beta$-catenin signalling pathways.Oncol Rep 2017;38:36393649.

13 Li X, Yang L, Shuai T, Piao T, Wang R: MiR-433 inhibits retinoblastoma malignancy by suppressing Notch1 and PAX6 expression. Biomed Pharmacother 2016;82:247-255.

14 Wang XC, Ma Y, Meng PS, Han JL, Yu HY, Bi LJ: miR-433 inhibits oral squamous cell carcinoma (OSCC) cell growth and metastasis by targeting HDAC6.Oral Oncol 2015;51:674-682.

15 Song G, Wang L: Transcriptional mechanism for the paired miR-433-3p and miR-127 genes by nuclear receptors SHP and ERRgamma. Nucleic Acids Res 2008;36:5727-5735.

-16 Guo LH,Li H,Wang F, Jia Yu,Jin-Sheng He: The Tumor Suppressor Roles of miR-433-3p and miR-127 in Gastric Cancer. Int J Mol Sci 2013;14:14171-1484.

17 Zheng Q, Chen C, Guan H, Kang W, Yu C: Prognostic role of microRNAs in human gastrointestinal cancer:A systematic review and meta-analysis.Oncotarget. 2017;8:46611-46623.

18 Yang 0, Huang J, Lin S: Regulatory effects of miRNA on gastric cancer cells.Oncol Lett 2014;8:651-656. 